\title{
Hausdorff Dimension and mean porosity
}

\author{
Pekka Koskela*, Steffen Rohde** \\ Department of Mathematics, University of Michigan, Ann Arbor, MI 48109, USA
}

Received: 24 June 1995 / Revised version: 9 July 1996

\section{Introduction}

A recent result of Jones and Makarov [JM] states that the Hausdorff dimension (or the Minkowski dimension) of the boundary of the image of the disk under a uniformly $\alpha$-Hölder continuous univalent function does not exceed $2-C \alpha$, where $C$ is a universal constant. Moreover, they show that this statement is sharp in the sense that there exist constants $C$ for which this estimate fails even for small $\alpha$. The Hölder continuity of a univalent function is equivalent to an appropriate logarithmic growth condition on the hyperbolic metric of the image domain as was shown by Becker and Pommerenke [BP]. The hyperbolic metric is comparable to the quasihyperbolic metric (see Section 5 for the definition) in a simply connected plane domain by the Koebe distortion theorem, and hence the result of Jones and Makarov can be interpreted as a sharp dimension estimate for boundaries of simply connected plane domains that satisfy a logarithmic growth condition on their quasihyperbolic metric. This growth condition can be defined for any bounded domain in the Euclidean space $\mathbb{R}^{d}$. Smith and Stegenga [SS1] produced an estimate for the dimension in such a general situation by modifying the ideas of Jones and Makarov. However, their estimate is weaker than the result of Jones and Makarov when applied to the case of a simply connected plane domain.

The main purpose of this paper is to establish sharp dimension estimates for sets satisfying a certain porosity condition. Roughly speaking we require that

\footnotetext{
* Current address: Department of Mathematics, University of Jyväskylä, P.O. Box 35, FIN-40351 Jyväskylä, Finland (e-mail: pkoskela@math.jyu.fi)

** Current address: Department of Mathematics, Technical University of Berlin, Strasse des 17. Juni 135, D-10623 Berlin, Germany (e-mail: rohde@math.tu-berlin.de)

The first author is partially supported by the NSF and by the Finnish Academy. Part of this research was done when the second author was supported as a Feodor Lynen fellow of the Alexander von Humboldt foundation. The authors also acknowledge support through the HCM network "Conformal geometry and geometric function theory".
} 
each point of a set $E$ can be approached through the complement of $E$ by a sequence of balls with radii comparable to the distance to this point, together with an estimate for the "density" of this sequence. See Section 2 for the actual definition. It is well known and easy to establish that if the balls in such a sequence appear in all scales, then a bound on the Hausdorff (or Minkowski) dimension for $E$ follows. A growth condition on the hyperbolic metric of the complement of $E$ does not guarantee that one could find these balls in all scales and hence the situation is more complicated. Our definition for porosity tackles this problem; we assume that the balls are evenly distributed in an averaged sense which then allows us to apply our estimates to the situations considered by Jones and Makarov, and Smith and Stegenga. As a special case of our results we recover the estimate of Jones and Makarov. Our notion of porosity and the results of this paper have very recently found applications in complex dynamics, see [PR].

The paper is organized as follows. We introduce the porosity condition in Section 2 and prove there the basic dimension estimate. Section 3 contains applications to the boundary behavior of conformal and quasiconformal mappings. In Section 4 we prove a result related to a theorem of Gerasch. Section 5 contains a geometric application and is independent from Sections 3 and 4.

We would like to thank the referee for his comments.

\section{Mean porous sets}

We recall that the Minkowski dimension of a set $E \subset \mathbb{R}^{d}$ is defined by

$$
\operatorname{dim}_{M}(E) \equiv \inf \left\{\delta: \limsup _{r \rightarrow 0} M_{\delta}(E, r)<\infty\right\},
$$

where, for $0<\delta$ and $0<r, M_{\delta}(E, r) \equiv \inf \left\{k r^{\delta}: F \subset \bigcup_{1}^{k} B\left(x_{i}, r\right)\right\}$. It follows that the Minkowski dimension of $E$ is greater or equal to the usual Hausdorff dimension $\operatorname{dim}_{H}(E)$ of $E$, with equality for sufficiently regular sets $E$.

Recall that a set $E \subset \mathbb{R}^{d}$ is said to be porous if there is a constant $c$ so that for each $x \in E$ and all $r>0$ the ball $B(x, r)$ contains a point $y$ with $d(y, E) \geq c r$. Here and in the sequel $d(y, E)$ denotes the distance of $y$ to $E$. It is well known that

$$
\operatorname{dim}_{M}(E) \leq d-C c^{d}
$$

for each bounded porous set $E$, where $c$ is the constant associated with $E$ and $C$ depends only on $d$. For this see e.g. [MV],[T]. This bound for the dimension of $E$ in terms of $c$ is sharp, see the discussion following the proof of Theorem 2.1 below. Porous sets have also been called $K$-sets, see $[\mathrm{Br}]$ and references therein. For a survey on various porosity conditions in the one dimensional situation see [Za].

We introduce the concept of mean porosity and produce estimates for the dimension of sets that are mean porous. It is easy to see that sets $E$ whose points can be approached by a twisted cone from the complement of $E$ are mean porous. 
Moreover, in Section 5, we show that the boundary of a domain that satisfies a logarithmic growth condition on its quasihyperbolic metric is mean porous.

Let $E \subset \mathbb{R}^{d}$ be a bounded set and $0<\varepsilon, c \leq 1$. Denote by $A_{n}(x)$ the annulus $A_{n}(x)=\left\{y \in \mathbb{R}^{d}:(1+\varepsilon)^{-n}<|x-y|<(1+\varepsilon)^{-n+1}\right\}$ and set

$$
\chi_{n}(x)=\left\{\begin{array}{l}
1 \text { if } A_{n}(x) \text { contains } y \text { with } d(y, E)>c \varepsilon|x-y| \\
0 \text { otherwise } .
\end{array}\right.
$$

Notice that the width of the annulus $A_{n}(x)$ is $\varepsilon(1+\varepsilon)^{-n}$. Set

$$
s_{n}(x)=\sum_{k=1}^{n} \chi_{k}(x)
$$

We say that $E$ is mean $\varepsilon$-porous if there is an integer $n_{0}$ such that

$$
\frac{s_{n}(x)}{n}>\frac{1}{2}
$$

for each $x \in E$ and all $n \geq n_{0}$.

Next, $E$ is weakly mean $\varepsilon$-porous, if

$$
\liminf _{n \rightarrow \infty} \frac{s_{n}(x)}{n}>\frac{1}{2}
$$

for each $x \in E$.

Note that our definitions of mean porosity involve the two parameters $c$ and $\varepsilon$ rather than just one. A more precise notation would be $s_{n}(x ; E, \varepsilon, c)$, which we shall use only in Proposition 3.1 in Section 3. Similarly, the dimension of mean porous sets depends on both parameters, as described in Theorem 2.1 below. However, in the applications we have in mind the parameter $c$ will be kept away from 0 and plays a technical role only. Therefore our notation supresses this dependence.

It is easy to see that $E$ is mean $\varepsilon$-porous if $E$ is. Also notice that a mean porous set has empty interior. Porous sets are mean porous with appropriate parameters: If $E$ is $c_{1}$ - porous, then $E$ is mean porous with parameters $\varepsilon=1 / 2$ and $c=C c_{1}$. On the other hand, it is not hard to give examples of mean porous sets that fail to be porous.

The main result of this section is the following theorem.

Theorem 2.1. If $E \subset \mathbb{R}^{d}$ is bounded and mean $\varepsilon$-porous, then

$$
\operatorname{dim}_{M}(E) \leq d-C \varepsilon^{d-1} .
$$

If $E$ is weakly mean $\varepsilon$-porous, the same estimate holds for Hausdorff-dimension instead of Minkowski dimension. The constant $C$ depends on $d$ and $c$ only and satisfies

$$
C \geq C(d) c^{d}
$$


Remarks. These estimates are sharp in the sense that the exponents are best possible, as we shall show by an example following the proof of the theorem. The use of the Marcinkiewicz integrals as in [JM] would easily give (2.2) with $d-1$ replaced by any exponent $\alpha>d-1$ but $C$ replaced by $C_{\alpha}$. Our proof of (2.2) does not use Marcinkiewicz integrals directly, but follows arguments of Zygmund [Zy]. If $E$ is $c$ - porous, we reobtain (setting $\varepsilon=1 / 2$ ) the sharp bound $\operatorname{dim}_{M}(E) \leq d-C c^{d}$ mentioned earlier. The constant $1 / 2$ in (2.1) could be replaced by any other positive number less than 1 . Only the constant $C$ in Theorem 2.1 would be affected by this third parameter.

For the proof of Theorem 2.1 we record a well known consequence (see e.g. [Bo]) of the Hardy-Littlewood maximal theorem:

Lemma 2.2. If $\mathscr{B}$ is a family of balls in $\mathbb{R}^{d}$ and $p>0$, then

$$
\int_{\mathbb{R}^{d}}\left(\sum_{B \in \mathscr{B}} \chi_{B / p}(x)\right)^{k} d x \leq\left(\frac{C_{1} k}{p^{d}}\right)^{k} \int_{\mathbb{R}^{d}}\left(\sum_{B \in \mathscr{B}} \chi_{B}(x)\right)^{k} d x
$$

for all $k \geq 1$, where $C_{1}=C_{1}(d)$.

Here and in what follows, $c B$ and $B / p$ denote balls with the same center as $B$ but of radii $c$ and $1 / p$ times that of $B$. For the convenience of the reader let us briefly sketch the proof of Lemma 2.2. First of all, the case $k=1$ is immediate. Using duality it thus suffices to verify that

$$
\int_{\mathbb{R}^{d}}\left|u_{p} \psi\right| d x \leq \frac{C_{1} k}{p^{d}}\left(\int_{\mathbb{R}^{d}}|u|^{k} d x\right)^{1 / k}\left(\int_{\mathbb{R}^{d}}|\psi|^{k /(k-1)} d x\right)^{(k-1) / k}
$$

whenever $\psi \in C_{0}^{\infty}\left(\mathbb{R}^{d}\right), u_{p}, u$ are the two sums from the claim, and $k>1$. This is accomplished by replacing $\psi$ by its maximal function and using the boundedness of the maximal operator on $L^{k /(k-1)}\left(\mathbb{R}^{d}\right)$; the constant $C_{1} k / p^{d}$ then arises from the norm of the maximal operator and from volume comparison between $B$ and $B / p$ when we introduce the maximal function.

Proof of Theorem 2.1. We can assume that $E$ is a compact set contained in the unit cube $Q_{0}$ and that $n_{0}=1$ (otherwise decompose $E$ into finitely many sets $E_{j}$ such that some translate of $E_{j}$ has the above properties). Throughout the proof, $E+t$ denotes the $t-$ neighborhood of $E$ and $|E+t|$ is the Lebesgue measure of $E+t$.

First we shall construct a family $\mathscr{B}$ of pairwise disjoint open balls $B \subset E+1$ with the property that for every positive integer $j$ and every $x \in E+2^{-j}$,

$$
\sum_{B \in \mathscr{B}} \chi_{c_{0} /(c \varepsilon) B}(x) \geq c_{1}^{j}
$$

where $c_{0}, c_{1}$ depend on $d$ only.

Let $\mathscr{W}$ be a Whitney decomposition of $2 Q_{0} \backslash E$. We refer the reader to [S] for the construction and basic properties of such a decomposition; the important fact is that $\mathscr{W}$ consists of pairwise disjoint cubes whose diameters are comparable 
to distance to the boundary of $2 Q_{0} \backslash E$. For every cube $Q \in \mathscr{W}$ we consider its Whitney decomposition into cubes $Q_{l}=Q_{l}(Q), l=1,2,3, \cdots$. Denote the largest ball contained in $Q_{l}$ by $B_{l}=B_{l}(Q)$. Let $\mathscr{B}=\left\{B_{l}(Q): Q \in \mathscr{W}, l=1,2, \ldots\right\}$ be the collection of all these balls.

Let $j$ be a positive integer and $x \in E+2^{-j}$. Choose $x^{\prime} \in E$ with $\left|x-x^{\prime}\right|<2^{-j}$ and consider an index $k$ with $\chi_{k}\left(x^{\prime}\right)=1$. Thus the annulus $A_{k}\left(x^{\prime}\right)$ contains a point $y$ with $d(y, E)>c \varepsilon\left|x^{\prime}-y\right|$. Let $Q \in \mathscr{W}$ be the cube containing $y$; then

$$
\operatorname{diam}(Q) \geq c \varepsilon_{4}^{1}(1+\varepsilon)^{-k}
$$

and $A_{k}$ is not entirely contained in $Q$ because $x^{\prime} \in E$. Pick a cube $Q_{l} \subset A_{k}\left(x^{\prime}\right)$ of maximal diameter from the Whitney decomposition of $Q$ and let $B_{l}$ be the ball associated with $Q_{l}$. Then $B_{l} \subset A_{k}\left(x^{\prime}\right)$, and

$$
\operatorname{diam}\left(B_{l}\right) \geq c_{2} c \varepsilon(1+\varepsilon)^{-k},
$$

where $c_{2}$ depends only on $d$. If $(1+\varepsilon)^{-k}>2^{-j}$ we conclude $x \in c_{0} /(c \varepsilon) B_{l}$ with $c_{0}=c_{0}(d)$. But $(1+\varepsilon)^{-k}>2^{-j}$ provided $k<{ }_{\varepsilon}^{j} \log 2$, as $\log (1+\varepsilon) \leq \varepsilon$ for $0<\varepsilon$. Hence (2.3) follows from (2.1) and (2.4). The construction of the family $\mathscr{B}$ is complete.

We define $p=c \varepsilon$ and claim that

$$
\left|E+2^{-j}\right| \leq c_{3}|E+1| 2^{-c_{4} j c p^{d-1}}
$$

for all $j \geq 0$ with constants $c_{3}, c_{4}$ depending on $d$ only. As in [MV], this inequality easily implies (2.2) (use the Besicovitch covering theorem [Zi][1.3.5]) as well as the desired estimate for $C$.

It suffices to show that, for some constant $\gamma$,

$$
\int_{E+2-j} \exp \left(\gamma p^{d} \sum_{B \in \mathscr{B}} \chi_{c_{0} / p B}(x)\right) d x \leq c_{3}|E+1|
$$

because

$$
\exp \left(\gamma p^{d} \sum \chi_{c_{0} / p B}(x)\right) \geq \exp \left(\gamma p^{d} c_{1} \frac{j}{\varepsilon}\right)=\exp \left(\gamma c_{1} j c p^{d-1}\right)
$$

on $E+2^{-j}$.

Write

$$
u(x)=\gamma p^{d} \sum_{B \in \mathscr{B}} \chi_{c_{0} / p B}(x) .
$$

Then

$$
\begin{gathered}
\int_{E+2^{-j}} \exp (u(x)) d x \leq \int_{E+1} \exp (u(x)) d x \leq \sum_{k \geq 0} \int_{E+1} u(x)^{k} d x \\
\leq|E+1|+\sum_{k>0} \int_{\mathbb{R}^{d}} u(x)^{k} d x
\end{gathered}
$$




$$
\leq|E+1|+\sum_{k>0} \frac{\left(\gamma p^{d}\right)^{k}}{k !} \int_{\mathbb{R}^{d}}\left(\sum_{B \in \mathscr{B}} \chi_{c_{0} / p B}(x)\right)^{k} d x .
$$

Using Lemma 2.2 we obtain

$$
\begin{aligned}
\int_{E+2^{-j}} \exp (u(x)) d x & \leq|E+1|+\sum_{k>0} \frac{\left(\gamma p^{d}\right)^{k}}{k !}\left(\frac{C_{1} k}{p^{d}}\right)^{k} \int_{\mathbb{R}^{d}}\left(\sum_{B \in \mathscr{B}} \chi_{B}(x)\right)^{k} d x \\
& \leq|E+1|\left(1+\sum_{k>0} \frac{\left(C_{1} \gamma k\right)^{k}}{k !}\right) .
\end{aligned}
$$

As the last series converges for $\gamma<1 /\left(C_{1} e\right)$ we have proven (2.5) and therefore (2.2) follows.

To finish the proof of Theorem 2.1, note that weakly mean $\varepsilon$ - porous sets are countable unions of bounded mean $\varepsilon$ - porous sets. Thus

$$
\operatorname{dim}_{H}(E) \leq d-C \varepsilon^{d-1}
$$

follows from (2.2).

An example. We shall give an example showing the sharpness of Theorem 2.1. Roughly speaking, we construct a set by removing an $\varepsilon$ - neighborhood of the coordinate axes in the unit cube and by repeating this process on all dyadic subcubes. To apply the estimate [McM][Proposition 2.2] of the Hausdorff dimension of Cantor-like sets, finite collections $E_{k}$ of closed sets in $\mathbb{R}^{d}$ will be constructed, having the basic properties

(a) for distinct elements $F_{1}, F_{2}$ of $E_{k},\left|F_{1} \cap F_{2}\right|=0$ (again $|$.$| denotes Lebesgue$ measure in $\mathbb{R}^{d}$ ),

(b) every $F \in E_{k+1}$ is contained in some $F^{\prime} \in E_{k}$,

(c) every $F \in E_{k}$ contains at least one element of $E_{k+1}$.

There are two parameters that control the dimension of $E=\bigcap_{k} \bigcup_{F \in E_{k}} F$ : If $0<\delta<1$ is such that $\operatorname{diam}(F) \leq c \delta^{k}$ for all $F \in E_{k}$ and if $\Delta$ is a lower bound for the density of $E_{k+1}$ in $E_{k}$, i.e. $\left|\bigcup_{F \in E_{k+1}} F \cap F^{\prime}\right| \geq \Delta\left|F^{\prime}\right|$ for all $F^{\prime} \in E_{k}$, then

$$
\operatorname{dim}_{H}(E) \geq d-|\log \Delta| /|\log \delta|
$$

by [McM][Proposition 2.2]. In our situation, the elements of $E_{k}$ will not necessarily be disjoint as required in $[\mathrm{McM}]$, but the assumption (a) is enough for the proof of (2.6).

Let $\varepsilon$ be of the form $\varepsilon=2^{-n}$ for some integer $n>2$. Let $Q_{0} \subset \mathbb{R}^{d}$ be the cube

$$
Q_{0}=\left\{\left(x_{1}, \ldots, x_{d}\right):-1 \leq x_{j} \leq 1\right\}
$$

and define a set $\hat{Q}_{0} \subset Q_{0}$ by removing the $\varepsilon$-neighborhood (in the sup-norm) of the coordinate axes from $Q_{0}$. Thus

$$
\hat{Q}_{0}=\left\{\left(x_{1}, \ldots, x_{d}\right) \in Q_{0}:\left|x_{j}\right| \geq \varepsilon \text { for at least two indexes } j\right\} .
$$


$E_{0}$ consists of $\hat{Q}_{0}$ alone. To obtain $E_{k}$ from $E_{k-1}$, subdivide $Q_{0}$ into $2^{d k}$ dyadic cubes $Q_{k}^{j}$ of sidelength $2^{-k+1}$ in the natural way. Define subsets $\hat{Q}_{k}^{j} \subset Q_{k}^{j}$ by taking a similarity $T: Q_{0} \rightarrow Q_{k}^{j}$ and setting $\hat{Q}_{k}^{j}=T\left(\hat{Q}_{0}\right)$. Then

$$
E_{k}=\left\{F \cap \hat{Q}_{k}^{j}: F \in E_{k-1}, j=1, \ldots, 2^{d k}\right\} .
$$

Thus for every $F \in E_{k}, F \neq \emptyset$, there is a cube $Q_{k}$ containing $F$, and every cube $Q_{k}$ contains at most one element of $E_{k}$. Thus (a) and (b) are satisfied. It is not hard to see that every (nonempty) $F \in E_{k}$ contains a dyadic cube $Q_{k+3}^{j}$. Not only does this imply (c), but it also yields an estimate of $\Delta$ : As $\left|Q_{0} \backslash \hat{Q}_{0}\right| \leq d \varepsilon^{d-1} 2^{d}$, we have $\left|\bigcup_{F \in E_{k+1}} F \cap F^{\prime}\right| \geq\left|F^{\prime}\right|-2^{-d k+d} d \varepsilon^{d-1} \geq\left(1-d 2^{3 d} \varepsilon^{d-1}\right)\left|F^{\prime}\right|$ for all $F^{\prime} \in E_{k}$. With $\Delta=1-d 2^{3 d} \varepsilon^{d-1}$ and $\delta=1 / 2, \operatorname{dim}_{H}(E) \geq d-c_{d} \varepsilon^{d-1}$ follows from (2.6). Notice that $E$ is mean $\varepsilon$-porous: For every $d$ there is a constant $r_{d}$ so that every $(d-1)$ - sphere in $\mathbb{R}^{d}$ of radius at least $r_{d}$ contains a point with all but at most one coordinate being an integer. Thus, choosing the constant $c$ small enough (but independent of $\varepsilon$ ) we can achieve $s_{n}(x, E, \varepsilon, c)=n$ for all $x \in E$ and all $n$. This finishes the proof of the theorem.

The same construction can be used to produce porous sets $E$ of Hausdorff dimension at least $d-C c^{d}$ as described in the beginning of this section. The only modification is in the definition of $\hat{Q}_{0}$. Instead of removing a neighborhood of the axis from $Q_{0}$, one removes only the $\varepsilon$ - neighborhood of the origin. We leave the details to the reader.

Let us next give a simple but instructive application of Theorem 2.1. We say that a domain $D$ is a $c$-John domain if there is a distinguished point $x_{0}$ such that for any $x \in D$ we can find a curve $\gamma:[0, l] \rightarrow D$, parametrized by arclength and with $\gamma(0)=x, \gamma(l)=x_{0}$, and $d(\gamma(t), \partial D) \geq{ }_{c}^{1} t$ for each $0 \leq t \leq l$. For example, the usual snowflake domain is a $c$-John domain with some $c$. It is immediate that every $c$-John domain is mean $c^{-1}$ - porous. Hence we obtain the following corollary to Theorem 2.1.

Corollary 2.3. Let $D \subset \mathbb{R}^{d}$ be a c-John domain. Then

$$
\operatorname{dim}_{M}(\partial D) \leq d-C c^{1-d},
$$

where $C$ depends only on $d$.

The observation that the dimension of the boundary of a $c$-John domain is bounded away from $d$ is not new. However, the estimates given in [MV, SS1, T] seem to be no better than $d-C c^{-d}$. This weaker estimate is obtained by viewing the boundary of a $k$-John domain as a (mean) $\varepsilon$ - porous set, taking the constant $c$ of our definition of mean porosity proportional to $1 / k$ and setting $\varepsilon=1$. Then (2.2) together with the estimate of $C$ in Theorem 2.2 yields the weaker bound. We see that we gain in the exponent because we did not consider dyadic annuli, counting only "one hole" in each annulus, but subdivided the dyadic annuli, thus recognizing "more holes". 


\section{Hölder continuity}

The connection between Hölder continuity and the concept of mean porosity considered in Section 2 comes from the following observation.

Proposition 3.1. Let $f: B^{d} \rightarrow G \subset \mathbb{R}^{d}$ be a $K$ - quasiconformal map, normalized by $d\left(f(0), \partial f\left(B^{d}\right)\right)=1$. If $w \in S^{d-1}, 0<\varepsilon<1$ and $a>0$ are such that

$$
|f(t w)-f(w)| \leq a(1-t)^{\varepsilon}
$$

for all $0 \leq t<1$, then

$$
s_{n}\left(f(w) ; \partial f\left(B^{d}\right), \varepsilon, c\right) \geq \frac{3}{4} n-c^{\prime} .
$$

The number $c$ depends on $K$ and $d$ only, and $c^{\prime}$ depends on $K, d, \varepsilon$ and $a$.

This estimate immediately shows that the boundary of the quasiconformal image of $B^{d}$ is mean $\varepsilon$ - porous, provided the quasiconformal map is Hölder continuous with exponent $\varepsilon$.

By combining Proposition 3.1 with Theorem 2.1 we arrive at the following corollary that extends the analogous result of Jones and Makarov [JM] for the case of conformal mappings of the disk in the plane.

Corollary 3.2. Let $f: B^{d} \rightarrow G \subset \mathbb{R}^{d}$ be $K$ - quasiconformal and suppose

$$
\left|f(x)-f\left(x^{\prime}\right)\right| \leq M\left|x-x^{\prime}\right|^{\varepsilon}
$$

for all $x, x^{\prime} \in B^{d}$. Then

$$
\operatorname{dim}_{M}(\partial G) \leq d-c(d, K) \varepsilon^{d-1} .
$$

Let us point out that the constant $c(d, K)$ in Corollary 3.2 really depends on $K$. Indeed, for each $1 / 2<\varepsilon<1$ there is a quasiconformal mapping $f$ of the plane that satisfies the above Hölder condition with exponent $\varepsilon$ and maps the unit circle onto a snowflake curve whose Hausdorff dimension equals $1 / \varepsilon$. For the existence of such a mapping see e.g. [K2].

We postpone the proof of Proposition 3.1 and continue with further applications. Notice that bounded quasiconformal maps always satisfy the Hölder continuity estimate of Proposition 3.1 on many radii:

Proposition 3.3. Let $f$ be a bounded quasiconformal map of $B^{d}$. Fix $0<\lambda \leq$ $d-1$. Then for $H_{\lambda}$-almost every $w \in S^{d-1}$ the radial limit $f(w)$ exists and

$$
|f(w)-f(t w)| \leq M_{w}(1-t)^{\lambda / d} .
$$


Here $H_{\lambda}$ stands for $\lambda$ - dimensional Hausdorff measure. Proposition 3.3 can be found in [K1]. For the convenience of the reader we sketch a simple proof below directly after the proof of Proposition 3.1.

If $B \subset S^{d-1}$ denotes the set of points where we have the (radial) Hölder continuity estimate of Proposition 3.1 with exponent $\alpha>0$ and $A$ is the complement, then Proposition 3.3 says $\operatorname{dim}_{H} A \leq d \alpha$, whereas Proposition 3.1 implies that $f(B)$ is weakly mean $\alpha$-porous. Thus Theorem 2.1 yields the following corollary that gives a quantitative version of the main theorem in the paper [K1] of the first author and that extends Theorem B.2 in the paper [JM] by Jones and Makarov.

Corollary 3.4. For every $d \geq 2$ and $K \geq 1$ there is a constant $c(d, K)>0$ such that the following holds: If $f: B^{d} \rightarrow \mathbb{R}^{d}$ is $K$ - quasiconformal, then for every $0<\alpha<d-1$ there is a partition $S^{d-1}=A \cup B$ with

$$
\begin{aligned}
& \operatorname{dim}_{H} A \leq \alpha \quad \text { and } \\
& \operatorname{dim}_{H} f(B) \leq d-c \alpha^{d-1} .
\end{aligned}
$$

Proof of Proposition 3.1. Fix $0<c<1$, to be determined later. Consider an index $j<n$ with $\chi_{j}(f(w))=0$. Thus $d(y, \partial G) \leq c \varepsilon(1+\varepsilon)^{-j+1}$ for all $y \in A_{j}(f(w))$. The curve $\gamma=f([0, w])$ intersects the two boundary components of $A_{j}(f(w))$ in two points $a=f\left(t_{a} w\right)$ and $b=f\left(t_{b} w\right)$, say. Thus the quasihyperbolic distance $k_{G}(a, b)$ of $a$ and $b$ in $G$ is at least $1 / c(1+\varepsilon)$; see Section 5 for the definition and some properties of the quasihyperbolic distance. As quasiconformal maps are quasi-isometries for large distances in the quasihyperbolic metrics (see [GO, p. 62]), the quasihyperbolic distance $k_{B^{d}}\left(t_{a} w, t_{b} w\right)$ is bounded from below by $C / c$ provided $c$ is small enough. Here $C$ depends on $K$ and $d$.

Consider the largest $t<1$ with

$$
|f(t w)-f(w)|=(1+\varepsilon)^{-n} .
$$

Then

$$
\log \frac{1}{1-t}=k_{B^{d}}(0, t w) \geq \sum k_{B^{d}}\left(t_{a} w, t_{b} w\right) \geq\left(n-s_{n}\right) \frac{C}{c},
$$

where the summation is over all $j$ with $\chi_{j}(f(w))=0$. On the other hand,

$$
(1+\varepsilon)^{-n}=|f(t w)-f(w)| \leq a(1-t)^{\varepsilon}
$$

and thus

$$
\log \frac{1}{1-t} \leq \frac{n}{\varepsilon} \log (1+\varepsilon)+\log \left(a^{1 / \varepsilon}\right)
$$

We conclude that

$$
n \geq \frac{C}{c}\left(n-s_{n}\right)-a^{\prime}
$$

and the proposition follows by choosing $c$ small enough.

Proof of Proposition 3.3. For $j=1,2,3 \cdots$, define

$$
E(j)=\left\{x \in S^{d-1}: \limsup _{r \rightarrow 0} r^{-\lambda} \int_{B(w, r) \cap B^{d}} J_{f}(x) d x \geq j^{-1}\right\} .
$$


Given $\delta>0$, pick for each $w \in E(j)$ a radius $0<r_{w}<\delta$ such that

$$
r_{w}^{-\lambda} \int_{B\left(w, r_{w}\right) \cap B^{d}} J_{f}(x) d x \geq 1 /(2 j) .
$$

By the Besicovitch covering theorem $[\mathrm{Zi}, 1.3 .5]$ we find a sequence $\left(w_{i}\right)$ (possibly finite) so that

$$
E_{j} \subset \cup B\left(w_{i}, r_{i}\right)
$$

and for each $x$

$$
\sum_{i} \chi_{B\left(w_{i}, r_{i}\right)}(x) \leq C
$$

where $C$ depends only on $d$. Thus

$$
\begin{gathered}
\sum_{i} r_{i}^{\lambda} \leq(2 j)^{\lambda} \sum_{i} \int_{B\left(w_{i}, r_{i}\right) \cap B^{d}} J_{f}(x) d x \\
\leq C(2 j)^{\lambda} \int_{\cup B\left(w_{i}, r_{i}\right) \cap B^{d}} J_{f}(x) d x \leq C(2 j)^{\lambda} \int_{B^{d} \backslash B(0,1-\delta)} J_{f}(x) d x .
\end{gathered}
$$

Letting $\delta$ go to zero, we conclude that $H_{\lambda}\left(E_{j}\right)=0$, as desired.

Let now $w \in S^{d-1}$ be such that

$$
\int_{B(w, r) \cap B^{d}} J_{f}(x) d x \leq r^{\lambda}
$$

for all sufficiently small $r$; this is then true for $H_{\lambda}$-almost every $w$. Write $B_{t}=$ $B(t w,(1-t) / 2), 0<t<1$. Then by quasiconformality of $f$ and the above estimate we conclude for all $t$ sufficiently close to 1 that

$$
\operatorname{diam}\left(f B_{t}\right)^{d} \leq C\left|f B_{t}\right| \leq C \int_{B_{t}} J_{f}(x) d x \leq C\left(\frac{3}{2}(1-t)\right)^{\lambda} .
$$

Thus the oscillation of $f$ in $B_{t}$ is at most a constant times $\operatorname{diam}\left(B_{t}\right)^{\lambda / d}$. Using a chain of balls $B_{t}$ for which $1-t$ decreases in a geometric fashion, we conclude that $f$ has a radial limit $f(w)$ and for some constant $M$

$$
|f(w)-f(t w)| \leq M(1-t)^{\lambda / d} .
$$

\section{Around a theorem of Gerasch}

A theorem of Gerasch [G] says that

$$
\limsup _{x \rightarrow w} \frac{d\left(f(x), \partial f\left(B^{2}\right)\right)}{|f(x)-f(w)|}=1
$$

for almost every $w \in S^{1}$ and every conformal map $f$ of the unit disk. It has been shown by Martio and Näkki [MN] that the same result holds for quasiconformal maps in plane and space; in higher dimensions one naturally replaces $S^{1}$ by $S^{n-1}$ and $B^{2}$ by $B^{n}$. The next result is an extension from $n-1$ dimensional measure to Hausdorff measure. 
Theorem 4.1. Let $f$ be a $K$-quasiconformal map of $B^{d}$, and suppose $0<q<1$. Then for $H_{\lambda}$-almost every $w \in S^{d-1}$ the radial limit $f(w)$ exists and

$$
\limsup _{x \rightarrow w} \frac{d\left(f(x), \partial f\left(B^{d}\right)\right)}{|f(x)-f(w)|} \geq q .
$$

The estimates

$$
\lambda \leq \lambda(K, d, q)<d-1
$$

and

$$
\lambda(K, d, q) \rightarrow 0 \quad \text { as } q \rightarrow 0
$$

hold, the latter for all fixed $K$ and $d$.

Remark. The proof will show that

$$
\lambda \leq \frac{c(d, K)}{\log (1 / q)}
$$

for small $q$, and this is the best possible bound that our method of proof allows, except for the constants. Propositions 3.1 and 3.3 yield much better estimates for small $q$, namely $\lambda \leq c(d, K) q$. On the other hand, they do not yield nontrivial bounds for $q$ near 1 . The reason is that radial Hölder continuity of exponent $\alpha$ is only known to imply

$$
\limsup _{x \rightarrow w} \frac{d\left(f(x), \partial f\left(B^{d}\right)\right)}{|f(x)-f(w)|} \geq \alpha / 2
$$

in the conformal case, see [NP, p. 178]. Allowing unrestricted approach in Theorem 4.1 is what makes our proof work. It has been asked by C. Bishop [Bi] whether Gerasch's theorem holds with radial instead of unrestricted approach.

The next result would already follow from Propositions 3.1 and 3.3.

Corollary 4.2. Let $f$ be a $K$-quasiconformal map of $B^{d}$. Let $A \subset \partial f\left(B^{d}\right)$ consist of those points $x$ that cannot be approached by a sequence of balls contained in $f\left(B^{d}\right)$ whose radii are comparable to their distance to $x$. Then $\operatorname{dim} f^{-1}(A)=0$. This is in particular true for the set of density points of Lebesgue d-measure of $\partial\left(f\left(B^{d}\right)\right)$.

Notice that the theorem of Gerasch implies the result of Øksendal [Ø1, Ø2] on the support of harmonic measure in simply connected domains. Heinonen and Martio [HM] were first to prove that Øksendal's result holds for quasiconformal mappings of the disk, i.e., for each quasiconformal mapping $f$ of the disk there exists a set $E$ of full length on the boundary $T$ of the disk with the area of $f(T \backslash E)$ being zero. Their proof was based on estimates for the elliptic equation associated with $f$. There are by now several different proofs for this fact in any dimension, see the papers [H] by Hajlasz, [K1] by Koskela, and [MM] by Maly and Martio. Theorem 4.1 and Corollary 4.2 provide us with an argument that adds to this list. 
The remainder of this section is devoted to the proof of Theorem 4.1. The proof rests on an estimate for modulus of curve families that we state as Proposition 4.3. This estimate could be tracked down in the literature with some work by going through estimates for various capacities. We give a complete and simple proof below for the convenience of the reader.

Proposition 4.3. Let $u$ be a non-negative Borel function in $B^{d}$ such that for a set $E \subset S^{d-1}$

$$
\int_{\gamma} u d s \geq 1
$$

for each rectifiable curve $\gamma$ joining $E$ to $B(0,1 / 2)$ in $B^{d}$. Then for each $\alpha>0$

$$
\int_{B^{d}} u^{d} d x \geq c_{d} \alpha^{d-1} H_{\alpha}^{\infty}(E) .
$$

Here $H_{\alpha}^{\infty}(E)=\inf \left\{\sum_{i} r_{i}^{\alpha}: E \subset \bigcup_{i} B\left(x_{i}, r_{i}\right)\right\}$ is the usual Hausdorff content of E.

Proof. Fix $\alpha>0$. To simplify our notation we assume that $u$ is extended as zero to $\mathbb{R}^{d} \backslash B^{d}$. Fix $y \in E$ and let $0<r<R<1$. Then for each $w \in S^{d-1}$ we have by the Hölder inequality

$$
\begin{gathered}
\int_{r}^{R} u(y+t w) d t=\int_{r}^{R} u(y+t w) t^{(d-1) / d} t^{(1-d) / d} d t \\
\leq\left(\int_{r}^{R} u(y+t w)^{d} t^{d-1} d t\right)^{1 / d}\left(\log \frac{R}{r}\right)^{(d-1) / d} .
\end{gathered}
$$

Set $r_{j}={ }_{j^{p}}, p$ to be determined later. For $j=1,2, \ldots$ write $A_{j}(y)=B\left(y, r_{j}\right) \backslash$ $B\left(y, r_{j+1}\right)$ and set $I_{j}(w)=\left(\int_{r}^{R} u(y+t w) d t\right)^{d}$, where $R=r_{j}, r=r_{j+1}$. Then integration of the above inequality with respect to $w$ over $S^{d-1}$ (and using $\log (1+$ $x) \leq x$ ) gives

$$
\int_{S^{d-1}} I_{j}(w) d \sigma \leq\left(\begin{array}{l}
p \\
j
\end{array}\right)^{d-1} \int_{A_{j}(y)} u^{d}(x) d x
$$

Suppose now that for each $j$

$$
\int_{A_{j}(y)} u^{d}(x) d x \leq c_{d} \frac{\alpha^{d-1}}{j^{p \alpha}},
$$

where the dimensional constant $c_{d}$ will be chosen later. Write $\operatorname{Bad}_{j}(s)=\{w \in$ $\left.S^{d-1}: I_{j}(w) \geq s\right\}$. Then

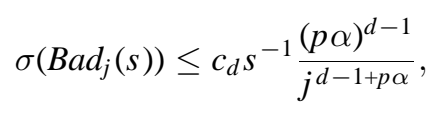

and

$$
\sigma\left(\cup_{j} \operatorname{Bad}_{j}\left(c_{d}^{\prime} j^{-2 d}\right)\right) \leq \sum_{j} c_{d} \frac{j^{2 d}(p \alpha)^{d-1}}{c_{d}^{\prime} j^{d-1+p \alpha}}=(p \alpha)^{d-1} \frac{c_{d}}{c_{d}^{\prime}} \sum_{j} \frac{1}{j^{p \alpha-d-1}}
$$


This can be made arbitrarily small by first choosing $p$ such that $p \alpha=d+3$ and then choosing $c_{d}$ appropriately (after choosing $c_{d}^{\prime}$ later).

For each $w \in S^{d-1}$ not in $\cup_{j} \operatorname{Bad}_{j}\left(c_{d}^{\prime} j^{-2 d}\right)$ we have

$$
\int_{0}^{1} u(y+t w) d t=\sum_{j} I_{j}(y)^{1 / d} \leq \sum_{j}\left(c_{d}^{\prime} \frac{1}{j^{2 d}}\right)^{1 / d},
$$

which is $<1$ if $c_{d}^{\prime}$ is chosen small enough. By the argument in the previous paragraph, there is some $w$ outside the bad set such that the segment of length 1 in $w$ direction from $y$ intersects $B(0,1 / 2)$. We conclude that there is an index $j$ such that

$$
\int_{A_{j}(y)} u^{d} d x \geq c_{d} \frac{\alpha^{d-1}}{j^{p \alpha}} .
$$

By the Besicovitch covering theorem we may then cover $E$ with balls $B\left(y_{j}, r_{n_{j}}\right)$ of the above type and so that only a bounded number (depending on $d$ ) of these balls overlap. Then

$$
\sum_{j} r_{n_{j}}^{\alpha}=\sum_{j}\left(1 / n_{j}\right)^{p \alpha} \leq c_{d}^{\prime \prime} \alpha^{1-d} \int_{B^{d}} u^{d} d x
$$

as desired.

Proof of Theorem 4.1. It is more convenient to work with the half space $\mathbb{R}_{+}^{d}=$ $\mathbb{R}^{d-1} \times \mathbb{R}_{+}$instead of the ball $B^{d}$. Given a $K$-quasiconformal map $f: \mathbb{R}_{+}^{d} \rightarrow$ $G \subset \mathbb{R}^{d}$ and $0<q<1$, let $E \subset \mathbb{R}^{d-1}=\partial \mathbb{R}_{+}^{d}$ be the set of those points $y$ for which $f(y, 0)$ exists as a radial limit (which happens $H_{\lambda}$-a.e. for every $\lambda>0$, see for instance $[\mathrm{Zo}]$ ) and furthermore

$$
\limsup _{(x, t) \rightarrow(y, 0)} \frac{d(f(x, t), \partial G)}{|f(x, t)-f(y, 0)|}<q
$$

holds. We want to show $H_{\lambda}(E)=0$ for some $\lambda<d-1$. Set $h_{\lambda}(E)=\inf \left\{\sum_{i} l_{i}^{\lambda}\right.$ : $\left.E \subset \bigcup_{i} Q_{i}\right\}$, where the $Q_{i}$ are dyadic cubes of sidelength $l_{i}$. Since $1 / c H_{\lambda}^{\infty} \leq$ $h_{\lambda} \leq c H_{\lambda}^{\infty}$ it is clear that $h_{\lambda}(E)=0$ if and only if $H_{\lambda}(E)=0$.

Consider

$$
E_{n}=\left\{y \in \mathbb{R}^{d-1}: \frac{d(f(x, t), \partial G)}{|f(x, t)-f(y, 0)|}<q \text { for all } x \in \mathbb{R}^{d-1} \text { and } 0<t<1 / n\right\} .
$$

If there is $c<1$ such that

$$
h_{\lambda}\left(E_{n} \cap Q\right) \leq c l^{\lambda}
$$

for all (dyadic) cubes $Q \subset \mathbb{R}^{d-1}$ of sidelength $l<1 / n$, then $h_{\lambda}\left(E_{n}\right)=0$ and thus $h_{\lambda}(E)=0$ follows easily.

By rescaling (i.e. composing $f$ with a similarity) it is enough to establish the existence of $c<1$ with (4.2) for the unit cube $Q_{0} \subset \mathbb{R}^{d-1}$ and $n=1$.

We distinguish two cases as to whether $q$ is small or close to one. 
If $q<q_{0}(d, K)$ (to be determined later), let $\Gamma$ be the family of those curves in $\mathbb{R}_{+}^{d}$ joining $E_{1} \cap Q_{0}$ to $B\left(e_{d+1}, 1 / 2\right), e_{d+1}$ denoting the $d+1-$ st unit vector. By standard distortion estimates for quasiconformal maps (or by compactness) and comparison to a spherical annulus we find

$$
M(\Gamma) \leq K M(f(\Gamma)) \leq c(d, K)\left(\log \frac{1}{q}\right)^{1-d} .
$$

Applying a Möbius transformation of $\mathbb{R}_{+}^{d}$ to $B^{d}$ (sending $e_{d+1}$ to 0 ) we obtain from Proposition 4.3

$$
h_{\lambda}\left(E_{1} \cap Q_{0}\right) \leq c H_{\lambda}^{\infty}\left(E_{1} \cap Q_{0}\right) \leq c(d, K)\left(\lambda \log \frac{1}{q}\right)^{1-d}
$$

and thus (4.2) with $\lambda \equiv c^{\prime}(d, K) / \log (1 / q)$. Thus the theorem is proven in the case $\lambda<d-1$. This determines $q_{0}$.

Now let $q_{0}(d, K) \leq q<1$. We may assume $d\left(f\left(e_{n+1}\right), \partial G\right)=1$. Standard modulus arguments (for example [HK,Lemma 6.6]) or compactness show that

$$
\min \left\{d(f(x, t), \partial G): x \in \frac{1}{4} Q_{0}\right\}<\phi(K, d, t) \rightarrow 0
$$

as $t \rightarrow 0$. For each $t>0$, pick $\left(x_{0}, t\right)$ realizing the minimum and let $\gamma$ be the preimage of a line segment joining $f\left(x_{0}, t\right)$ to a closest boundary point. Then $\operatorname{diam}(f(\gamma))<\phi(K, d, t)$, and hence the modulus of the curve family joining $f(\gamma)$ to $f\left(B\left(e_{n+1}, 1 / 2\right)\right)$ does not exceed $\psi(K, d, t) \rightarrow 0$ as $t \rightarrow 0$. Using the quasiconformality of $f$ we conclude that the diameter of $\gamma$ goes to zero as $t$ tends to zero. Hence the endpoint $y_{0}$ of $\gamma$ is contained in ${ }_{2}^{1} Q_{0}$ whenever $t$ is sufficiently small (depending only on $K$ and $d$ ).

It is standard to show that $\gamma$ lies in a cone (of opening depending on $d$ and $K$ only) around the ray $\left\{\left(y_{0}, t\right): t>0\right\}$. Let $\left(x_{1}, t_{1}\right) \in \gamma$ be so that $d\left(f\left(x_{1}, t_{1}\right), \partial G\right)=\varepsilon d\left(f\left(x_{0}, t_{0}\right), \partial G\right)$, where $\varepsilon<1$ is fixed later. Distortion estimates (or compactness) show $t_{1} \geq c\left(\varepsilon, k_{0}\right)=c(\varepsilon, d, K)>0$. For $y \in E_{1}$ we have

$$
\begin{aligned}
\left|f\left(x_{1}, t_{1}\right)-f(y, 0)\right| & \geq\left|f\left(x_{0}, t_{0}\right)-f(y, 0)\right|-\left|f\left(x_{1}, t_{1}\right)-f\left(x_{0}, t_{0}\right)\right| \\
& >\frac{1}{q} d\left(f\left(x_{0}, t_{0}\right), \partial G\right)-(1-\varepsilon) d\left(f\left(x_{0}, t_{0}\right), \partial G\right)
\end{aligned}
$$

and obtain

$$
d\left(f\left(x_{1}, t_{1}\right), \partial G\right)<q^{\prime}\left|f\left(x_{1}, t_{1}\right)-f(y, 0)\right|
$$

with $q^{\prime}=\varepsilon /(\varepsilon+(1-q) / q)$.

If $Q$ is the dyadic cube of sidelength $l$ satisfying $l \leq t_{1}<2 l$ and $x_{1} \in Q$, we conclude from (4.3) (or rather the proof of (4.3)) by rescaling

$$
h_{\lambda}\left(E_{1} \cap Q\right) \leq c(d, K)\left(\lambda \log \frac{1}{q^{\prime}}\right)^{1-d} l^{\lambda} .
$$

Choosing $\varepsilon=\varepsilon(q, d, K)$ small enough we obtain 


$$
h_{\frac{1}{2}}\left(E_{1} \cap Q\right)<\frac{1}{2} l^{1 / 2}
$$

and $Q \subset Q_{0}$, the latter because of the nontangential approach of $\gamma$. Consider the decomposition of $Q_{0}$ into $2^{d-1} l^{-(d-1)}$ dyadic cubes of sidelength $l$. As

$$
h_{\lambda}\left(E_{1} \cap Q\right) \leq l^{\lambda-\frac{1}{2}} h_{\frac{1}{2}}\left(E_{1} \cap Q\right) \leq \frac{1}{2} l^{\lambda}
$$

for $1 / 2 \leq \lambda \leq d-1$, we get

$$
h_{\lambda}\left(E_{1} \cap Q_{0}\right) \leq\left(2^{d-1} l^{-(d-1)}-1\right) l^{\lambda}+\frac{1}{2} l^{\lambda} .
$$

This implies the existence of $\lambda=\lambda(q, d, K)<d-1$ with $h_{\lambda}\left(E_{1} \cap Q_{0}\right) \leq c 2^{\lambda}$ for some universal $c<1$. We have shown (4.2) and the proof of the theorem is finished.

\section{Quasihyperbolic metric}

Let $D$ be a proper subdomain of $\mathbb{R}^{d}$. Then the quasihyperbolic distance of a pair $x, y$ of points in $D$ is defined to be the infimum of

$$
\int_{\gamma_{x, y}} d(\gamma(t), \partial D)
$$

where the infimum is taken over all rectifiable curves joining $x, y$ in $D$. We denote the quasihyperbolic distance of $x, y$ by $k_{D}(x, y)$. It is known (c.f. [GO]) that $x, y$ can be joined by a quasihyperbolic geodesic.

Following Gehring and Martio [GM] we say that $D$ satisfies a quasihyperbolic boundary condition if

$$
k_{D}\left(x, x_{0}\right) \leq \frac{1}{\varepsilon} \log \frac{1}{d(x, \partial D)}+C
$$

for a fixed $x_{0} \in D$ and for all $x \in D$. It then follows that $D$ is bounded and that any point in $D$ could act as the point $x_{0}$ (with possibly a different constant $C$ ). The constant $\varepsilon$ is the crucial one whereas $C$ plays a minor role.

If $D$ is a simply connected plane domain, then $k_{D}$ can be replaced by the hyperbolic metric provided we replace ${ }_{\varepsilon}^{1}$ by ${ }_{\varepsilon}^{2}$. Becker and Pommerenke [BP] have shown that a simply connected domain $D$ in the plane satisfies (5.1) with $k_{D}$ replaced by the hyperbolic metric if and only if $D$ is the image of the disk under a conformal mapping that is uniformly Hölder continuous with exponent $\varepsilon$. For a quasiconformal analog of this see [GM].

Using ideas of Jones and Makarov [JM], Smith and Stegenga [SS2] established that

$$
\operatorname{dim}_{M}(\partial D) \leq d-C \varepsilon^{d+1}
$$

with a constant $C$ depending only on $d$ provided (5.1) holds. For simply connected planar domains $D$ the stronger estimate $\operatorname{dim}_{M}(\partial D) \leq d-C \varepsilon$ holds by the work of Jones and Makarov [JM]. We prove a sharp extension of this result as a consequence of Theorem 2.1. 
Theorem 5.1. Suppose that $D \subset \mathbb{R}^{d}$ satisfies the quasihyperbolic boundary condition (5.1). Then

$$
\operatorname{dim}_{M}(\partial D) \leq d-C \varepsilon^{d-1}
$$

with a constant $C$ depending only on $d$.

Proof. By Theorem 2.1 it suffices to show that $\partial D$ is mean $\varepsilon$-porous. Also, we may assume that $0<\varepsilon \leq 1$ as a simple computation verifies that (5.1) never holds with $\varepsilon>1$, and we may assume that $d\left(x_{0}, \partial D\right) \geq 1$. Fix $x \in \partial D$ and pick a positive integer $n$. Select a point $y \in B\left(x,(1+\varepsilon)^{-n}-(1+\varepsilon)^{-2 n}\right) \cap D$. Let $\gamma$ be a quasihyperbolic geodesic joining $y$ to $x_{0}$. It follows from (5.1) that

$$
k_{D}\left(w, x_{0}\right) \leq \frac{1}{\varepsilon} \log \frac{1}{l(w, y)}+C_{1}
$$

for each point $w$ on the geodesic, where $l(w, y)$ is the length of the part of the geodesic joining $y$ to $w$ and the constant $C_{1}$ depends only on $\varepsilon$ and $C$, see [SS1, Theorem 3]. Select $w$ so that $l(w, y)=(1+\varepsilon)^{-2 n}$. Then

$$
k_{D}\left(w, x_{0}\right) \leq \frac{2 n}{\varepsilon} \log (1+\varepsilon)+C_{1} \leq 2 n+C_{1} .
$$

Moreover, $w \in B\left(x,(1+\varepsilon)^{-n}\right)$, and by taking $c=1 / 10$, we see that the subcurves of $\gamma$ in those $A_{k}(x), 1 \leq k \leq n$, for which $\chi_{k}(x)=0$, contribute to the line integral over $\gamma$ by at least

$$
10 \geq 5
$$

Hence

$$
k_{D}\left(w, x_{0}\right) \geq 5\left(n-s_{n}(x)\right),
$$

and we conclude that $s_{n}(x) \geq n / 2$ whenever $n$ is sufficiently large (depending only on $C_{1}$ and $\varepsilon$ ). The claim follows from Theorem 2.1.

\section{References}

[BP] J. Becker, C. Pommerenke: Hölder continuity of conformal mappings and non-quasiconformal Jordan curves, Comment. Math. Helv. 57 (1982), 221-225.

[Bi] C. Bishop: How geodesics approach the boundary in a simply connected domain, J. Analyse Math. 64 (1994), 291-325.

[Bo] B. Bojarski: Remarks on Sobolev imbedding inequalities, Lecture Notes in Math. 1351, Berlin, Heidelberg, New York: Springer 1989, 52-68.

[Br] J. Bruna: Boundary interpolation sets for holomorphic functions smooth to the boundary and BMO, Trans. Amer. Math. Soc. 264 (1981), 393-409.

[GM] F.W. Gehring, O. Martio: Lipschitz classes and quasiconformal mappings, Ann. Acad. Sci. Fenn. Ser. A I Math. 10 (1985), 203-219.

[GO] F.W. Gehring, B. Osgood: Uniform domains and the quasihyperbolic metric, J. Analyse Math. 36 (1979), 50-74.

[G] T.E. Gerasch: On the accessibility of the boundary of a simply connected domain, Michigan Math. J. 33 (1986), 201-207.

[H] P. Hajłasz: Boundary behavior of Sobolev mappings, Proc. Amer. Math. Soc. 123 (1995), $1145-1148$. 
[HK] J. Heinonen, P. Koskela: The boundary distortion of a quasiconformal mapping, Pacific J. Math. 165 (1994), 93-114.

[HM] J. Heinonen, O. Martio: Estimates for $F$-harmonic measures and $\emptyset$ ksendal's theorem for quasiconformal mappings, Indiana Univ. Math. J. 36 (1987), 659-683.

[JM] P.W. Jones, N.G. Makarov: Density properties of harmonic measure, Ann. Math. 142 ( 199)5 427-455.

[K1] P. Koskela: The radial behavior of a quasiconformal mapping, Duke Math. J. 74 (1994), 667-679.

[K2] P. Koskela: The degree of regularity of a quasiconformal mapping, Proc. Amer. Math. Soc. 122 (1994), 769-772.

[M] N.G. Makarov: On the distortion of boundary sets under conformal mappings, Proc. London Math. Soc. 51 (1985), 369-384.

[McM] C. McMullen: Area and Hausdorff dimension of Julia sets of entire functions, Trans. Amer. Math. Soc. 300 (1987), 329-342.

[MM] J. Maly, O. Martio: Lusin's condition (N) and mappings of the class $W^{1, n}$, J. Reine Angew. Mat. 458 (1995), 19-36.

[MN] O. Martio, R. Näkki: Boundary accessibility of a domain quasiconformally equivalent to a ball, preprint

[MV] O. Martio, M. Vuorinen: Whitney cubes, $p$-capacity, and Minkowski content, Expo. Math. 5 (1987), 17-40.

[NP] R. Näkki, B. Palka: Boundary angles, cusps and conformal mappings, Complex Variables 22 (1981), 221-224.

[Ø1] B. Øksendal: Null sets for measures orthogonal to $R(X)$, Amer. J. Math. 94 (1972), 331-342.

[O2] B. Øksendal: Brownian motion and sets of harmonic measure zero, Pacific J. Math. 95 (1981), 179-192.

[PR] F. Przytycki, S. Rohde: Porosity of Collet-Eckmann Julia sets, preprint

[SS1] W. Smith, D.A. Stegenga: Hölder domains and Poincaré domains, Trans. Amer. Math. Soc. 319 (1990), 67-100.

[SS2] W. Smith, D.A. Stegenga: Exponential integrability of the quasihyperbolic metric in Hölder domains, Ann. Acad. Sci. Fenn. Ser. A I Math. 16 (1991), 345-360.

[S] E. Stein: Singular integrals and differentiability properties of functions, Princeton: Princeton University Press, 1970

[T] D.A. Trotsenko: Properties of regions with nonsmooth boundary, Sibirsk. Mat. Zh. 22 (1981), 221-224. (Russian)

[Za] L. Zajicek: Porosity and $\sigma$-porosity, Real Anal. Exchange 13 (1987/88), 314-350.

[Zi] W.P. Ziemer: Weakly differentiable functions, Graduate Texts in Mathematics, 120, 1. Berlin, Heidelberg, New York: Spinger 1989

[Zo] V.A. Zorič: Angular boundary values of quasiconformal mappings of a ball, Dokl. Akad. Nauk. SSSR 179 (1967), 1492-1494.

[Zy] A. Zygmund: On certain Lemmas of Marcinkiewicz and Carleson, J. Approximation Theory 2 (1969), 249-257. 